\title{
ETP-Mine: An Efficient Method for Mining Transitional Patterns
}

\author{
B. Kiran Kumar ${ }^{1}$ and A. Bhaskar ${ }^{2}$ \\ ${ }^{1}$ Department of M.C.A., Kakatiya Institute of Technology \& Science, A.P. INDIA. \\ ${ }^{2}$ Department of M.C.A., Kakatiya Institute of Technology \& Science, A.P. INDIA. \\ bhaskar_adepueyahoo.com
}

\begin{abstract}
A Transaction database contains a set of transactions along with items and their associated timestamps. Transitional patterns are the patterns which specify the dynamic behavior of frequent patterns in a transaction database. To discover transitional patterns and their significant milestones, first we have to extract all frequent patterns and their supports using any frequent pattern generation algorithm. These frequent patterns are used in the generation of transitional patterns. The existing algorithm (TP-Mine) generates frequent patterns, some of which cannot be used in generation of transitional patterns. In this paper, we propose a modification to the existing algorithm, which prunes the candidate items to be used in the generation of frequent patterns. This method drastically reduces the number of frequent patterns which are used in discovering transitional patterns. Extensive simulation test is done to evaluate the proposed method.
\end{abstract}

\section{KEYWORDS}

Frequent Patterns, Association Rules, Transitional Patterns, Significant milestones.

\section{INTRODUCTION}

Frequent pattern mining was extensively studied in the area of data mining research. These frequent patterns are used in many different data mining tasks like Association rule mining [2] [3] [12], sequential pattern mining [4], structured pattern mining [7], correlation mining [5], and associative classification [9]. Many algorithms were proposed to find frequent patterns in transaction databases namely Apriori [3], FP-growth [8], and Eclat [15]. But these methods generate large number of patterns when the minimum support threshold is low. Out of these patterns most are not required in data mining tasks. To avoid these useless or redundant patterns, new types of patterns were introduced, namely maximal frequent itemsets [1] [6] [11], and closed frequent itemsets [10].

All the above methods do not consider time stamps associated with the transactions. So, the above methods do not reveal the dynamic behavior of the patterns. For example, in an electronics shop database, the sales for Air conditioners will be high during the summer season and the sales will be low in other seasons. If we consider total transactions, Air conditioners will become frequent, but these are frequent in only summer season. To capture the dynamic behavior of the patterns, new type patterns were introduced, called Transitional patterns [13] [14]. Transitional patterns can be both positive and negative. Positive transitional patterns increase their frequency 
at some time stamp. Negative transitional patterns decrease their frequency at some time stamp. Significant milestone of the transitional pattern is the time point at which the frequency of the transitional pattern changes most frequently. TP-Mine algorithm [14] was proposed to discover the transitional patterns and their significant milestones.

The TP-Mine algorithm contains two major steps. In the first step all the frequent patterns are generated from the transaction database. In the second step these frequent patterns are used in generating transitional patterns and their significant milestones within the given milestone range that the user is interested. But, some of the frequent patterns generated in the first step are not used in the second step.

In this paper we are proposing a modification to the TP-Mine algorithm to eliminate the useless frequent patterns generated in the first step. Thereby we can reduce the number of computations required in generating transitional patterns.

The rest of the paper is organized as follows. Section 2 describes preliminaries and definitions used in TP-Mine algorithm. Section 3 describes TP-Mine Algorithm. In section 4, we describe the modified TP-Mine (ETP-Mine) algorithm. Section 5 presents experimental results of our work. Finally, in section 6 we conclude our work.

\section{PRELIMINARIES AND DEFINITIONS}

Mining frequent patterns is one of the fundamental operations in data mining applications for extracting interesting patterns from databases. Let $I=\left\{i_{l}, i_{2} \ldots i_{n}\right\}$ be a set of items. Let $\mathrm{D}$ be a set of database transactions where each transaction $\mathrm{T}$ is a set of items and $\|\mathrm{D}\|$ be the number of transactions in $\mathrm{D}$. Given $\mathrm{X}=\left\{\mathrm{i}_{j} \ldots \mathrm{i}_{k}\right\} \subseteq \mathrm{I}(\mathrm{j} \leq \mathrm{k}$ and $1 \leq \mathrm{j}, \mathrm{k} \leq \mathrm{n})$ is called a pattern. The support of a pattern $\mathrm{X}$ in $\mathrm{D}$ is the number of transactions in $\mathrm{D}$ that contains $X$. Pattern $X$ will be called frequent if its support is no less than a user specified minimum support threshold.

Definition 2.1: The cover of an itemset $\mathrm{X}$ in $\mathrm{D}$, denoted by $\operatorname{cov}(X, D)$, is the number of transactions in which the item $\mathrm{X}$ appears.

Definition 2.2: An itemset $\mathrm{X}$ in a transaction database $\mathrm{D}$ has a support, denoted by $\sup (X, D)$, which is the ratio between $\operatorname{cov}(X, D)$ to the number of transactions in D i.e., ID\|.

$$
\sup (X, D)=\frac{\operatorname{cov}(X, D)}{\|D\|}
$$

Definition 2.3: Assuming that the transactions in a transaction database D are ordered by their time-stamps, the position of a transaction $\mathrm{T}$ in $\mathrm{D}$, denoted by $\rho(\mathrm{T})$, is the number of transactions whose time-stamp is less than or equal to that of $T$. Thus $1 \leq \rho(T) \leq\|D\|$.

Definition 2.4: The $\mathrm{i}^{\text {th }}$ transaction of a pattern $\mathrm{X}$ in $\mathrm{D}$, denoted by $\mathrm{T}^{\mathrm{i}}(\mathrm{X})$, is the $i^{\text {th }}$ transaction in $\operatorname{cov}(X)$ with transactions ordered by their positions, where $1 \leq i \leq \operatorname{cov}(X, D)$.

Definition 2.5: The $\mathrm{i}^{\text {th }}$ milestone of a pattern $\mathrm{X}$ in $\mathrm{D}$, denoted by $\xi^{\mathrm{i}}(\mathrm{X})$, is defined as

$$
\xi^{\mathrm{i}}(\mathrm{X})=\frac{\rho(\operatorname{Ti}(\mathrm{X}))}{\|D\|} \times 100 \% \text { where } 1 \leq \mathrm{i} \leq \operatorname{cov}(X) .
$$

Definition 2.6: The support of the pattern $\mathrm{X}$ before its $\mathrm{i}^{\text {th }}$ milestone in $\mathrm{D}$, denoted by $\sup _{-}^{i}(X)$ is defined as 
International Journal of Database Management Systems ( IJDMS ) Vol.2, No.3, August 2010

$$
\sup _{-}^{i}(X)=\frac{i}{\rho(\operatorname{Ti}(\mathrm{X}))} \quad \text { where } 1 \leq \mathrm{i} \leq \operatorname{cov}(X)
$$

Definition 2.7: The support of the pattern $X$ after its $i^{\text {th }}$ milestone in $\mathrm{D}$, denoted by $\sup _{+}^{i}(X)$ is defined as

$$
\sup _{+}^{i}(X)=\frac{\operatorname{cov}(x)-i}{\|\mathrm{D}\|-\rho(\operatorname{Ti}(\mathrm{X}))} \quad \text { where } 1 \leq \mathrm{i} \leq \operatorname{cov}(X) .
$$

Definition 2.8: The Transitional ratio of the pattern $\mathrm{X}$ at its $\mathrm{i}^{\text {th }}$ milestone in $\mathrm{D}$ is defined as

$$
\operatorname{tran}^{i}(X)=\frac{\sup _{+}^{i}(X)-\sup _{-}^{i}(X)}{\operatorname{Max}\left(\sup _{+}^{i}(X), \sup _{-}^{i}(X)\right)} \quad \text { where } 1 \leq \mathrm{i} \leq \operatorname{cov}(X) .
$$

Definition 2.9: A pattern $\mathrm{X}$ is a transitional pattern (TP) in $\mathrm{D}$ if there exists at least one milestone of $\mathrm{X}, \xi^{\mathrm{k}}(\mathrm{X}) \in \mathrm{T}_{\xi}$, such that:

$$
\text { 1. } \sup _{-}^{k}(X) \geq t_{s} \text { and } \sup _{+}^{k}(X) \geq t_{s} \text { and } 2 .\left|\operatorname{tran}^{i}(X)\right| \geq t_{t}
$$

where $T_{\xi}$ is a range of $\xi^{i}(X)(1 \leq i \leq \operatorname{cov}(X)), t_{s}$ and $t_{t}$ are called pattern support threshold and transitional pattern threshold, respectively. $X$ is called a Positive Transitional Pattern $(P T P)$ when $\operatorname{tran}^{k}(X)>0$; and $X$ is called a Negative Transitional Pattern $(N T P)$ when $\operatorname{tran}^{k}(X)<0$.

Definition 2.10: The significant frequency-ascending milestone of a positive transitional pattern $\mathrm{X}$ with respect to a time period $\mathrm{T}_{\xi}$ is defined as a tuple, $\left(\xi^{\mathrm{M}}(\mathrm{X}), \operatorname{tran}^{\mathrm{M}}(\mathrm{X})\right)$, where $\xi^{\mathrm{M}}(\mathrm{X}) \in \mathrm{T}_{\xi}$ is the $\mathrm{M}^{\text {th }}$ milestone of $\mathrm{X}$ such that

$$
\text { 1. } \sup _{-}^{M}(X) \geq t_{s} ; \text { and } 2 . \forall \xi^{i}(X) \in T_{\xi}, \operatorname{tran}^{M}(X) \geq \operatorname{tran}^{i}(X)
$$

Definition 2.11: The significant frequency-descending milestone of a negative transitional pattern $\mathrm{X}$ with respect to a time period $\mathrm{T}_{\xi}$ is defined as a tuple, $\left(\xi^{\mathrm{N}}(\mathrm{X}), \operatorname{tran}^{\mathrm{N}}(\mathrm{X})\right)$, where $\xi^{\mathrm{N}}(\mathrm{X}) \in \mathrm{T}_{\xi}$ is the $\mathrm{N}^{\text {th }}$ milestone of $\mathrm{X}$ such that

\begin{tabular}{|c|c|c|}
\hline TID & List of Item IDs & Time stamp \\
\hline 001 & P1,P2,P3,P5 & Nov, 2005 \\
\hline 002 & $\mathrm{P} 1, \mathrm{P} 2$ & Dec, 2005 \\
\hline 003 & P1,P2,P3,P8 & Jan, 2006 \\
\hline 004 & P1,P2,P5 & Feb, 2006 \\
\hline 005 & $\mathrm{P} 1, \mathrm{P} 2, \mathrm{P} 4$ & Mar, 2006 \\
\hline 006 & P1,P2,P4,P5,P6 & Apr, 2006 \\
\hline 007 & P1,P2,P3,P4,P6 & May, 2006 \\
\hline 008 & P1,P4,P6 & Jun, 2006 \\
\hline 009 & P4,P5,P6 & Jul, 2006 \\
\hline 010 & P1,P2,P3,P4,P5,P6 & Aug, 2006 \\
\hline 011 & P1,P3,P4,P6 & Sep, 2006 \\
\hline 012 & P1,P3,P5 & Oct, 2006 \\
\hline 013 & P1,P2,P3,P6,P7 & Nov, 2006 \\
\hline 014 & P1,P3,P4,P5 & Dec, 2006 \\
\hline 015 & P1,P3,P4 & Jan, 2007 \\
\hline 016 & P1,P2,P3,P5 & Feb, 2007 \\
\hline
\end{tabular}

$$
\text { 1. } \sup _{+}^{N}(X) \geq t_{s} ; \text { and } 2 . \forall \xi^{i}(X) \in T_{\xi}, \operatorname{tran}^{N}(X) \leq \operatorname{tran}^{i}(X)
$$

\section{TP-MINE ALGORITHM}

Consider the following transaction database shown in Table 1.

Table 1: Example Database

TP-Mine algorithm [14] generates the set of positive and negative transitional patterns with their significant milestones. 
Input: A transaction database (D), milestone range $\left(\mathrm{T}_{\xi}\right)$, pattern support threshold $\left(\mathrm{t}_{\mathrm{s}}\right)$, and transitional pattern $\left(\mathrm{t}_{\mathrm{t}}\right)$.

We considered $\mathrm{D}$ as set of transactions listed in Table $1, \mathrm{~T}_{\xi}=\{25 \%, 75 \%\}, \mathrm{t}_{\mathrm{s}}=0.05, \mathrm{t}_{\mathrm{t}}=0.5$

This algorithm has two major phases. In the first phase all frequent itemsets along with their support counts are generated using Apriori [3] or FP-growth [8] with $t_{\mathrm{s}}$ as minimum support threshold. In this step, the algorithm generates $n$ number of frequent patterns whose support $\geq t_{s}$ from the transaction database $\mathrm{D}$. The resultant frequent patterns $(\mathrm{n}=87)$ are shown in Table 2.

Table 2: Set of frequent patterns

In the second phase, the algorithm computes the support counts $\left(c_{k}\right)$ of all the frequent patterns on the set from the first transaction to the transaction just before the time period $\mathrm{T}_{\xi}$. Then it computes

\begin{tabular}{|lc|lc|lc|lc|}
\hline FP & Sup & FP & Sup & FP & Sup & FP & Sup \\
\hline P1 & 15 & P3,P5 & 5 & P1,P6,P7 & 1 & P1,P2,P5,P6 & 2 \\
\hline P2 & 10 & P3,P6 & 4 & P2,P3,P4 & 2 & P1,P2,P6,P7 & 1 \\
\hline P3 & 10 & P3,P7 & 1 & P2,P3,P5 & 3 & P1,P3,P4,P5 & 2 \\
\hline P4 & 9 & P3,P8 & 1 & P2,P3,P6 & 3 & P1,P3,P4,P6 & 3 \\
\hline P5 & 8 & P4,P5 & 4 & P2,P3,P7 & 1 & P1,P3,P5,P6 & 1 \\
\hline P6 & 7 & P4,P6 & 6 & P2,P3,P8 & 1 & P1,P3,P6,P7 & 1 \\
\hline P7 & 1 & P5,P6 & 3 & P2,P4,P5 & 2 & P1,P4,P5,P6 & 2 \\
\hline P8 & 1 & P6,P7 & 1 & P2,P4,P6 & 3 & P2,P3,P4,P5 & 1 \\
\hline P1,P2 & 10 & P1,P2,P3 & 6 & P2,P5,P6 & 2 & P2,P3,P4,P6 & 2 \\
\hline P1,P3 & 10 & P1,P2,P4 & 4 & P2,P6,P7 & 1 & P2,P3,P5,P6 & 1 \\
\hline P1,P4 & 8 & P1,P2,P5 & 5 & P3,P4,P5 & 2 & P2,P3,P6,P7 & 1 \\
\hline P1,P5 & 7 & P1,P2,P6 & 4 & P3,P4,P6 & 3 & P2,P4,P5,P6 & 2 \\
\hline P1,P6 & 6 & P1,P2,P7 & 1 & P3,P5,P6 & 1 & P3,P4,P5,P6 & 1 \\
\hline P1,P7 & 1 & P1,P2,P8 & 1 & P3,P6,P7 & 1 & P1,P2,P3,P4,P5 & 1 \\
\hline P1,P8 & 1 & P1,P3,P4 & 5 & P4,P5,P6 & 3 & P1,P2,P3,P4,P6 & 2 \\
\hline P2,P3 & 6 & P1,P3,P5 & 5 & P1,P2,P3,P4 & 2 & P1,P2,P3,P5,P6 & 1 \\
\hline P2,P4 & 4 & P1,P3,P6 & 4 & P1,P2,P3,P5 & 3 & P1,P2,P3,P6,P7 & 1 \\
\hline P2,P5 & 5 & P1,P3,P7 & 1 & P1,P2,P3,P6 & 3 & P1,P2,P4,P5,P6 & 2 \\
\hline P2,P6 & 4 & P1,P3,P8 & 1 & P1,P2,P3,P7 & 1 & P1,P3,P4,P5,P6 & 1 \\
\hline P2,P7 & 1 & P1,P4,P5 & 3 & P1,P2,P3,P8 & 1 & P2,P3,P4,P5,P6 & 1 \\
\hline P2,P8 & 1 & P1,P4,P6 & 5 & P1,P2,P4,P5 & 2 & P1,P2,P3,P4,P5,P6 1 \\
\hline P3,P4 & 5 & P1,P5,P6 & 2 & P1,P2,P4,P6 & 3 & & \\
\hline
\end{tabular}

the milestones of $\mathrm{P}_{\mathrm{k}}(1 \leq \mathrm{k} \leq \mathrm{n})$ within the range $\mathrm{T}_{\xi}$. At each valid milestone $\xi^{c_{k}}\left(P_{k}\right)$ during the scan, it calculates the support of $\mathrm{P}_{\mathrm{k}}$ before $\xi^{c_{k}}\left(P_{k}\right)$ i.e., $\sup _{-}^{c_{k}}\left(P_{k}\right)$ and support of $\mathrm{P}_{\mathrm{k}}$ after $\xi^{c_{k}}\left(P_{k}\right)$ i.e., $\sup _{+}^{c_{k}}\left(P_{k}\right)$. If both of them are greater than $\mathrm{t}_{\mathrm{s}}$, the algorithm then checks the transitional ratio of $P_{k}$. If the ratio is greater than $t_{t}$ then $P_{k}$ is a positive transitional pattern. Then the algorithm checks whether the transitional ratio of $P_{k}$ is greater than the current maximal transitional ratio of $P_{k}$. If yes, the set of significant frequency ascending milestones of $\mathrm{P}_{\mathrm{k}}$ is set to contain $\left\{\xi^{c_{k}}\left(P_{k}\right), \operatorname{tran}^{c_{k}}\left(P_{k}\right)\right\}$ as its single element. If not but it is equal to the current maximal transitional ratio of $P_{k}$, $\left\{\xi^{c_{k}}\left(P_{k}\right), \operatorname{tran}^{c_{k}}\left(P_{k}\right)\right\}$ is added into significant frequency ascending milestone of $\mathrm{P}_{\mathrm{k}}$. Similarly it computes negative transitional patterns and their significant milestones if $\operatorname{tran}^{c_{k}}\left(P_{k}\right) \leq-\mathrm{t}_{\mathrm{t}}$. 
Table 3. Set of Positive transitional patterns and their ascending milestones.

\begin{tabular}{|c|c|}
\hline Pattern & Ascending Milestone \\
\hline P3 & aug2006(62.5\%), $60.000 \%$ \\
\hline $\mathrm{P} 4$ & mar2006(31.25\%), $72.500 \%$ \\
\hline P6 & apr2006(37.5\%), $72.222 \%$ \\
\hline P1,P3 & aug2006(62.5\%), $60.000 \%$ \\
\hline $\mathrm{P} 1, \mathrm{P} 4$ & mar2006(31.25\%), $68.571 \%$ \\
\hline P1,P6 & apr2006(37.5\%), $66.667 \%$ \\
\hline $\mathrm{P} 3, \mathrm{P} 4$ & may2006(43.75\%), $67.857 \%$ \\
\hline P3,P5 & aug2006(62.5\%), $60.000 \%$ \\
\hline P3,P6 & may2006(43.75\%), $57.143 \%$ \\
\hline P4,P6 & apr2006(37.5\%), $66.667 \%$ \\
\hline $\mathrm{P} 1, \mathrm{P} 3, \mathrm{P}$ & may2006(43.75\%), $67.857 \%$ \\
\hline $\mathrm{P} 1, \mathrm{P} 3, \mathrm{P}=$ & aug2006(62.5\%), $60.000 \%$ \\
\hline $\mathrm{P} 1, \mathrm{P} 3, \mathrm{Pe}$ & may2006(43.75\%), $57.143 \%$ \\
\hline $\mathrm{P} 1, \mathrm{P} 4, \mathrm{Pe}$ & apr2006(37.5\%), $58.333 \%$ \\
\hline
\end{tabular}

Table 4. Set of Negative transitional patterns and their descending milestones.

\begin{tabular}{|c|c|}
\hline Pattern & Descending Milestone \\
\hline P2 & may2006(43.75\%),-66.667 \\
\hline P6 & sep2006(68.75\%),-63.333\% \\
\hline P1,P2 & may2006(43.75\%),-66.667 \\
\hline P1,P6 & sep2006(68.75\%),-56.000\% \\
\hline P2,P4 & may2006(43.75\%),-74.074 \\
\hline P2,P5 & apr2006(37.5\%),-60.000\% \\
\hline P4,P6 & aug2006(62.5\%),-66.667\% \\
\hline P1,P2,P4 & may2006(43.75\%), -74.074 \\
\hline P1,P2,P5 & apr2006(37.5\%),-60.000\% \\
\hline P1,P4,P6 & aug2006(62.5\%),-58.333\% \\
\hline P2,P4,P6 & may2006(43.75\%),-61.111 \\
\hline P1,P2,P4,P & may2006(43.75\%),-61.111 \\
\hline
\end{tabular}

It was observed that the frequent patterns which contains items P7 or P8 (i.e.,24 patterns) are not used in the second phase of the algorithm, because these items are not contained in the transactions whose position satisfying $\mathrm{T}_{\xi}$. Therefore generating these 24 patterns in the first phase of the algorithm is useless. So, we are proposing a method for pruning these useless frequent patterns in the first phase of the algorithm.

\section{ETP-MINE (EFFICIENT TP-MINE) ALGORITHM}

In this section, we are presenting an Efficient TP-Mine algorithm which eliminates the useless frequent patterns. The algorithm efficiently generates positive and negative transitional patterns and their significant milestones with respect to a pattern support threshold and transitional pattern threshold. 
Algorithm: ETP-Mine

Input:

A transaction database (D), an appropriate milestone range that the user is interested $\left(\mathrm{T}_{\xi}\right)$, pattern support threshold $\left(\mathrm{t}_{\mathrm{s}}\right)$, and transitional pattern threshold $\left(\mathrm{t}_{\mathrm{t}}\right)$.

\section{Output:}

The set of transitional patterns $\left(\mathrm{S}_{\mathrm{PTP}}\right.$ and $\left.\mathrm{S}_{\mathrm{NTP}}\right)$ with their significant milestones.

\section{Method:}

1: Generate frequent 1-itemsets $\left(L_{1}\right)$ and their supports from $\mathrm{D}$ using the candidate 1-itemsets which appears in the transactions whose position satisfying $T_{\xi}$

2: Extract frequent patterns, $P_{1}, P_{2}, P_{3}, \ldots, P_{n}$, and their supports using $L_{1}$ with $\min \_\sup =t_{s}$.

3: Scan the transactions from the first transaction to the last transaction before $T_{\xi}$ to compute the support counts, $c_{k}(1 \geq \mathrm{k} \geq \mathrm{n})$, of all the $\mathrm{n}$ frequent patterns on this part of the database.

4: $S_{P T P}=\varnothing, S_{N T P}=\varnothing$

5: for all $\mathrm{k}=1$ to $\mathrm{n}$ do

6: $\operatorname{MaxTran}\left(P_{k}\right)=0, \operatorname{MinTran}\left(P_{k}\right)=0$

7: $S_{F A M}\left(P_{k}\right)=\varnothing, S_{F D M}\left(P_{k}\right)=\varnothing$

8: end for

9: for all transactions $T_{i}$ whose position satisfying $T_{\xi}$ do

$$
\begin{array}{lc}
\text { 10: } & \text { for } \mathrm{k}=1 \text { to } \mathrm{n} \text { do } \\
\text { 11: } & \text { if } T_{i} \supseteq P_{k} \text { then } \\
\text { 12: } & c_{k}=c_{k}+1 \\
\text { 13: } & \text { if } \sup _{-}^{c_{k}}\left(P_{k}\right) \geq t_{s} \text { and } \sup _{+}^{c_{k}}\left(P_{k}\right) \geq t_{s} \text { then } \\
\text { 14: } & \text { if } \operatorname{tran}^{c_{k}}\left(P_{k}\right) \geq t_{t} \text { then } \\
\text { 15: } & \text { if } P_{k} \notin S_{P T P} \text { then } \\
\text { 16: } & \text { Add } P_{k} \text { to } S_{P T P} \\
\text { 17: } & \text { end if } \\
\text { 18: } & \text { if } \operatorname{tran}^{c_{k}}\left(P_{k}\right)>\operatorname{MaxTran}\left(P_{k}\right) \text { then } \\
\text { 19: } & S_{F A M}\left(P_{k}\right)=\left\{\xi^{c_{k}}\left(P_{k}\right), \operatorname{tran}^{c_{k}}\left(P_{k}\right)\right\} \\
\text { 20: } & \operatorname{MaxTran}\left(P_{k}\right)=\operatorname{tran}^{c_{k}}\left(P_{k}\right) \\
\text { 21: } & \text { else if } \operatorname{tran}^{c_{k}}\left(P_{k}\right)=\operatorname{MaxTran}\left(P_{k}\right) \text { then } \\
\text { 22: } & \text { Add }\left\{\xi^{c_{k}}\left(P_{k}\right), \operatorname{tran}^{c_{k}}\left(P_{k}\right)\right\} \text { to } S_{F A M}\left(P_{k}\right) \\
\text { 23: } & \text { end if } \\
\text { 24: } & \text { else if } \operatorname{tran}^{c_{k}}\left(P_{k}\right) \leq-t_{t} \text { then } \\
\text { 25: } & \text { if } P_{k} \notin S_{N T P} \text { then } \\
\text { 26: } & \text { Add } P_{k} \text { to } S_{N T P} \\
\text { 27: } & \text { end if }
\end{array}
$$


28:

29:

$30:$

31:

32:

33:

34:

35:

36:

37: end for

38: end for

39: return $S_{P T P}$ and $S_{F A M}\left(P_{k}\right)$ for each $P_{k} \in S_{P T P}$

40: return $S_{N T P}$ and $S_{F D M}\left(P_{k}\right)$ for each $P_{k} \in S_{N T P}$

if $\operatorname{tran}^{c_{k}}\left(P_{k}\right)<\operatorname{MinTran}\left(P_{k}\right)$ then

$\operatorname{MinTran}\left(P_{k}\right)=\operatorname{tran}^{c_{k}}\left(P_{k}\right)$

end if end if end if

$$
S_{F D M}\left(P_{k}\right)=\left\{\xi^{c_{k}}\left(P_{k}\right), \operatorname{tran}^{c_{k}}\left(P_{k}\right)\right\}
$$

else if $\operatorname{tran}^{c_{k}}\left(P_{k}\right)=\operatorname{MinTran}\left(P_{k}\right)$ then

Add $\left\{\xi^{c_{k}}\left(P_{k}\right), \operatorname{tran}^{c_{k}}\left(P_{k}\right)\right\}$ to $S_{F D M}\left(P_{k}\right)$

By executing this algorithm on the transaction database given in the Table 1, the number of

\begin{tabular}{|lc|lc|lc|lc|lc|}
\hline FP & Sup & FP & Sup & FP & Sup & FP & Sup & FP & Sup \\
\hline P1 & 15 & P2,P5 & 5 & P1,P3,P5 & 5 & P3,P5,P6 & 1 & P2,P3,P4,P6 & 2 \\
\hline P2 & 10 & P2,P6 & 4 & P1,P3,P6 & 4 & P4,P5,P6 & 3 & P2,P3,P5,P6 & 1 \\
\hline P3 & 10 & P3,P4 & 5 & P1,P4,P5 & 3 & P1,P2,P3,P4 & 2 & P2,P4,P5,P6 & 2 \\
\hline P4 & 9 & P3,P5 & 5 & P1,P4,P6 & 5 & P1,P2,P3,P5 & 3 & P3,P4,P5,P6 & 1 \\
\hline P5 & 8 & P3,P6 & 4 & P1,P5,P6 & 2 & P1,P2,P3,P6 & 3 & P1,P2,P3,P4,P5 & 1 \\
\hline P6 & 7 & P4,P5 & 4 & P2,P3,P4 & 2 & P1,P2,P4,P5 & 2 & P1,P2,P3,P4,P6 & 2 \\
\hline P1,P2 & 10 & P4,P6 & 6 & P2,P3,P5 & 3 & P1,P2,P4,P6 & 3 & P1,P2,P3,P5,P6 & 1 \\
\hline P1,P3 & 10 & P5,P6 & 3 & P2,P3,P6 & 3 & P1,P2,P5,P6 & 2 & P1,P2,P4,P5,P6 & 2 \\
\hline P1,P4 & 8 & P1,P2,P3 & 6 & P2,P4,P5 & 2 & P1,P3,P4,P5 & 2 & P1,P3,P4,P5,P6 & 1 \\
\hline P1,P5 & 7 & P1,P2,P4 & 4 & P2,P4,P6 & 3 & P1,P3,P4,P6 & 3 & P2,P3,P4,P5,P6 & 1 \\
\hline P1,P6 & 6 & P1,P2,P5 & 5 & P2,P5,P6 & 2 & P1,P3,P5,P6 & 1 & P1,P2,P3,P4,P5,P6 & 1 \\
\hline P2,P3 & 6 & P1,P2,P6 & 4 & P3,P4,P5 & 2 & P1,P4,P5,P6 & 2 & & \\
P2,P4 & 4 & P1,P3,P4 & 5 & P3,P4,P6 & 3 & P2,P3,P4,P5 & 1 & & \\
\hline
\end{tabular}

frequent patterns generated is reduced to 63 , which are shown in Table 5 and the transitional patterns shown in Table 3 and Table 4 are generated(no change in the transitional patterns).

Table 5. Frequent Patterns generated using ETP-Mine algorithm

\section{EXPERIMENTAL RESULTS}

To demonstrate the efficiency of the ETP-Mine algorithm we have done experiments on synthetic data obtained from a Grocery shop with 25 transactions and 16 items. Table 6 shows the comparison of TP-Mine algorithm and our ETP-Mine algorithm against the Grocery data.

Table 6: Comparison of TP-Mine and ETP-Mine algorithms.

\begin{tabular}{|c|c|c|}
\hline & \multicolumn{2}{|c|}{$\begin{array}{c}\text { Number of frequent patterns gener: } \\
\text { in }\end{array}$} \\
\hline Min_su & TP-Mine & ETP-Mine \\
\hline 1 & 141 & 114 \\
\hline 2 & 79 & 76 \\
\hline
\end{tabular}




\section{CONCLUSIONS}

The existing TP-Mine algorithm generates more number of frequent patterns which are not useful in generating transitional patterns and their milestones. In this paper we presented a modification to the existing TP-Mine algorithm, which eliminates useless frequent patterns for generating transitional patterns. Our experimental results showed that the proposed algorithm is highly efficient and scalable.

\section{REFERENCES}

[1] R.C. Agarwal, C.C. Aggarwal, and V.V.V. Prasad, "Depth First Generation of Long Patterns," Proc. Sixth ACM SIGKDD Int'l Conf. Knowledge Discovery and Data Mining (KDD '00), pp. 108-118, 2000.

[2] R. Agrawal, T. Imielinski, and A. Swami, "Mining Association Rules between Sets of Items in Large Databases," Proc. 1993 ACM SIGMOD Int'l Conf. Management of Data (SIGMOD '93), pp.207-216, 1993.

[3] R. Agrawal and R. Srikant, "Fast Algorithms for Mining Association Rules," Proc. 20th Int'l Conf. Very Large Data Bases, pp. 487-499, 1994.

[4] R. Agrawal and R. Srikant, "Mining Sequential Patterns," Proc.11th Int'1 Conf. Data Eng., pp. 3-14, 1995.

[5] S. Brin, R. Motwani, and C. Silverstein, "Beyond Market Baskets: Generalizing Association Rules to Correlations," Proc. ACM SIGMOD Int'l Conf. Management of Data (SIGMOD '97), pp. 265-276, 1997.

[6] D. Burdick, M. Calimlim, J. Flannick, J. Gehrke, and T. Yiu, "Mafia: A Maximal Frequent Itemset Algorithm," IEEE Trans.Knowledge and Data Eng., vol. 17, no. 11, pp. 1490-1504, Nov. 2005.

[7] J. W. Han, J. Pei, and X. F.Yan, "From Sequential Pattern Mining to Structured Pattern Mining: A Pattern-Growth Approach,"J. Computer Science and Technology, vol. 19, no. 3, pp. 257-279, 2004.

[8] J.W. Han, J. Pei, Y. W. Yin, and R.Y. Mao, "Mining Frequent Patterns without Candidate Generation: A Frequent-Pattern Tree Approach," Data Mining and Knowledge Discovery, vol. 8, no. 1, pp. 53-87, 2004.

[9] B. Liu, W. Hsu, and Y.-M. Ma, "Integrating Classification and Association Rule Mining," Proc. Fourth ACM SIGKDD Int'l Conf.Knowledge Discovery and Data Mining (KDD 98), pp. 80-86, 1998.

[10] N. Pasquier, Y. Bastide, R. Taouil, and L. Lakhal, "Discovering Frequent Closed Itemsets for Association Rules," Proc. Seventh Int'l Conf. Database Theory (ICDT '99), pp. 398-416, 1999.

[11] R.J. Bayardo, Jr., "Efficiently Mining Long Patterns from Data-bases,” Proc. 1998 ACM SIGMOD Int'l Conf. Management of Data (SIGMOD '98), pp. 85-93, 1998.

[12] R. Srikant and R. Agrawal, "Mining Generalized Association Rules," Future Generation Computer Systems, vol. 13, nos. 2/3,pp. 161-180, 1997.

[13] Q. Wan and A. An, “Transitional Patterns and Their Significant Milestones," Proc. Seventh IEEE Int'l Conf. Data Mining, 2007.

[14] Q. Wan and A. An, "Discovering Transitional Patterns and Their Significant Milestones in Transaction Databases," IEEE Trans. on Knowledge and Data Engg., vol.21,No.12 pp.1692-1707, Dec 2009.

[15] M.J. Zaki and K. Gouda, "Fast Vertical Mining Using Diffsets," Proc. Ninth ACM SIGKDD Int'l Conf. Knowledge Discovery and Data Mining (KDD ’03), pp. 326-335, 2003. 
B. Kiran Kumar received M.C.A. from Kakatiya University in 1998, and he is pursuing M.Tech in Computer Science and Engg. in JNTU, Hyderabad. He is working as Associate Professor in the department of M.C.A. He delivered guest lectures in the field of Data mining at various engineering colleges. His research interest includes Data mining. He is a member of ISTE.

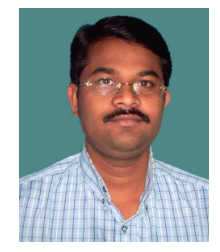

\begin{abstract}
A.Bhaskar received M.C.A. from Kakatiya University in 1998, and he is pursuing M.Tech in Computer Science and Engg. in JNTU, Hyderabad. He is working as Associate Professor in the department of M.C.A. He delivered guest lectures in the field of Artificial Intelligence at various engineering colleges. His research interest includes Data mining. He is a member of ISTE.
\end{abstract}

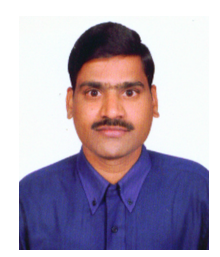

